\title{
Anabases
}

Grégory BONGARD-LEVINE, Corinne BONNET, Yuri LITVINENKO, Arnaldo MARCONE, Mongolus Syrio salutem optimam dat. La correspondance entre Mikhail Rostovtzeff et Franz Cumont

\section{Véronique Krings}

\section{OpenEdition}

\section{Édition électronique}

URL : http://journals.openedition.org/anabases/638

DOI : 10.4000/anabases.638

ISSN : 2256-9421

\section{Éditeur}

E.R.A.S.M.E.

\section{Édition imprimée}

Date de publication : 1 octobre 2009

Pagination : 283-284

ISSN : 1774-4296

\section{Référence électronique}

Véronique Krings, "Grégory bongard-LeVIne, Corinne bonnet, Yuri litVInenko, Arnaldo marcone, Mongolus Syrio salutem optimam dat. La correspondance entre Mikhail Rostovtzeff et Franz Cumont ", Anabases [En ligne], 10 | 2009, mis en ligne le 01 juillet 2011, consulté le 22 septembre 2020. URL : http:// journals.openedition.org/anabases/638; DOI : https://doi.org/10.4000/anabases.638

Ce document a été généré automatiquement le 22 septembre 2020

(c) Anabases 


\title{
Grégory BONGARD-LEVINE, Corinne BONNET, Yuri LITVINENKO, Arnaldo MARCONE, Mongolus Syrio salutem optimam dat. La correspondance entre Mikhail Rostovtzeff et Franz Cumont
}

\author{
Véronique Krings
}

\section{RÉFÉRENCE}

Grégory BONGARD-LEVINE, Corinne BONNET, Yuri LITVINENKo, Arnaldo MARConE, Mongolus Syrio salutem optimam dat. La correspondance entre Mikhail Rostovtzeff et Franz Cumont, Mémoires de l'Académie des Inscriptions et Belles-Lettres, t. XXXVI, Paris, Diffusion De Boccard, 2007, 364 p., $22 \mathrm{pl}$.

70 euros / ISBN 978-2-87754-193-0.

Entre 1897 et 1941, 164 lettres (conservées) ont été échangées par M. Rostovtzeff (1870-1952) et F. Cumont (1848-1947). Des 97 lettres de MR - conservées à l'Academia Belgica de Rome -, 8 seulement proviennent de la période russe et des premières années d'exil, avant l'arrivée à New Haven, en 1925. Des 67 lettres de FC - l'ensemble des lettres (sauf 2) proviennent de la Bibliothèque William Perkins de la Duke University -, seule une lettre datée de 1900 remonte à la période où il enseigne à l'Université de Gand, avant son exil volontaire à Rome et à Paris, en 1914. On constate deux pics de correspondance, le premier en 1928-30 avec 57 lettres et le second en 1937-39 avec 37 lettres. Les recherches sur les archives de MR ont débuté en 1988, celles qui portent sur la correspondance de FC en 1995. D'un travail mené en commun depuis 1997 par trois spécialistes du savant russe et une spécialiste du savant belge est issu, à côté de publications préliminaires, le présent ouvrage qui offre une édition annotée, 
par ordre chronologique, de la correspondance entre «deux authentiques monstres sacrés de l'historiographie de l'Antiquité, aussi différents que complémentaires » (p. 3). L'édition, dont les principes éditoriaux sont précisés à la p. XIII, est elle-même précédée d'une copieuse introduction qui privilégie trois axes. Celle-ci commence par une présentation des archives épistolaires, exposant les vides et les creux de la documentation (p. 3-5). Ensuite est retracée l'histoire des rapports entre les deux hommes, avec les grandes étapes de leurs échanges, dans le cadre de l'entre-deuxguerres (p. 6-9). L'histoire des fouilles de Doura-Europos (Salihiyé, sur l'Euphrate) (p. 10-48) constitue le plat de résistance de l'introduction. Le «dossier Doura » est aussi l'objet d'un appendice contenant des lettres d'autres correspondants et des pièces d'archives (actes de concession, rapports de fouilles, etc.) (p. 279-341) ainsi que d'un cahier rassemblant des documents photographiques anciens, certains se rapportant aux premières fouilles de $\mathrm{FC}$, et modernes (22 planches).

2 À partir de 1927, « l'aventure de Doura-Europos » constitue un thème majeur des lettres entre MR et FC; des fouilles sont menées conjointement par l'Académie des Inscriptions et Belles-Lettres et l'Université de Yale: FC et MR en sont les directeurs scientifiques, M. Pillet le directeur sur le terrain, remplacé par $\mathrm{C}$. Hopkins à partir de la cinquième campagne puis par F. Brown, pour les neuvième et dixième campagnes. Cet échange s'insère du reste, comme on le voit à travers l'ensemble de la correspondance de $\mathrm{FC}$, dans une « polyphonie » : plus de 400 lettres du fonds Cumont (sur 12 000) concernent Doura et, dans ce corpus, les lettres de MR ne représentent que $10 \%$ environ des échanges sur Doura. La correspondance s'avère d'autant plus précieuse que les matériaux des anciennes fouilles n'ont pas été totalement publiés et que les résultats de celles-ci, leurs méthodes et les conclusions des deux fouilleurs, ont fait l'objet de la part des archéologues et des historiens d'aujourd'hui, de critiques parfois sévères. Reconstruire, dans le détail, l'historique du chantier de Doura-Europos et remettre en perspective les interprétations historiques dont il a fait l'objet, tel a été l'objectif des éditeurs à travers la correspondance, qui de ce fait a conservé son importance, au même titre que les archives concernant l'exploration du site. D'une part, pour ce qui est du terrain, on a parfois reproché aux fouilleurs d'antan de s'être comportés en " amateurs » et d'avoir dirigé de loin le travail. Selon les éditeurs, la critique est fondée, mais excessive. D'autre part, Doura - et c'est sur ce point que l'apport des lettres apparaît avoir le plus retenu les éditeurs - a stimulé une féconde réflexion entre les deux savants. FC le premier a été chargé en 1922 par l'Académie des Inscriptions et Belles-Lettres - le site est alors sous le contrôle de l'armée française - de superviser une mission du corps expéditionnaire français sur le terrain. Les raisons qui ont amené MR à s'y intéresser à son tour sont multiples, comme on le voit à travers une lettre qu'il adresse à FC le 10 février 1925 ; l'attention qu'il porte à un parchemin de Doura publié par FC semble en être le déclencheur. De façon plus large, tous deux cherchent à comprendre les transformations majeures qui ont affecté le monde gréco-romain à la suite de son ouverture sur l'Orient, l'un en spécialiste de l'histoire religieuse, l'autre en historien de la vie économique et sociale et Doura-Europos, la "Pompéi syrienne " (MR), constitue à ce double titre un «terrain d'expérimentation historique idéal» (p. 18). Le croisement des lettres avec les publications des deux savants est à cet égard riche d'enseignements. Les dernières pages de l'introduction, consacrées aux autres thèmes de la publication (p. 48-53), nous plongent dans les passions communes des deux hommes - les trouvailles archéologiques, épigraphiques et papyrologiques et l'Orient hellénistique et romain - et dans les temps présents qui pèsent aussi sur 
l'écriture des travaux, en particulier l'ouvrage de MR, Social and Economic History of the Hellenistic World, paru à Oxford en 1941 et dédié à son épouse ainsi qu'à FC.

3 Voici une publication qui sera en outre précieuse pour ceux que retient l'étude des pratiques savantes et des réseaux scientifiques personnels et institutionnels, dans cet entre-deux-guerres qui a tant compté non seulement dans notre histoire mais aussi dans celle de nos disciplines. MR et FC en ont été non seulement des acteurs, mais aussi des témoins privilégiés. On ajoutera enfin que les auteurs de ce volume ont aussi participé à un livre tout fraîchement sorti de presse de J. Andreau et W. Berelowitch (éd.), Michel Ivanovitch Rostovtzeff, Bari, 2009, dont les contributions enrichiront encore celles du présent ouvrage.

\section{AUTEURS}

\section{VÉRONIQUE KRINGS}

Université de Toulouse (UTM)

vkrings@orange.fr 